\title{
SPHERES Tethered Formation Flight Testbed: Application to NASA's SPECS Mission
}

\author{
Soon-Jo Chung, Edmund M. Kong and David W. Miller \\ MIT Space Systems Laboratory, Cambridge, MA 02139
}

\begin{abstract}
This paper elaborates on theory and experiment of the formation flight control for the future space-borne tethered interferometers. The nonlinear equations of multi-vehicle tethered spacecraft system are derived by Lagrange equations and decoupling method. The preliminary analysis predicts unstable dynamics depending on the direction of the tether motor. The controllability analysis indicates that both array resizing and spin-up are fully controllable only by the reaction wheels and the tether motor, thereby eliminating the need for thrusters. Linear and nonlinear decentralized control techniques have been implemented into the tethered SPHERES testbed, and tested at the NASA MSFC's flat floor facility using two and three SPHERES configurations. The nonlinear control using feedback linearization technique performed successfully in both two SPHERES in-line configuration and three triangular configuration while varying the tether length. The relative metrology system, using the ultra sound metrology system and the inertial sensors as well as the decentralized nonlinear estimator, is developed to provide necessary state information.
\end{abstract}

Keywords: stellar interferometer, space tether, formation flight

\section{INTRODUCTION}

The quest for finer angular resolution in astronomy inevitably leads to larger apertures since the optical resolution is proportional to the wavelength over the diameter of a circular aperture. Unfortunately, the primary mirror diameter for space telescopes is limited by volume and mass constraints of current launch vehicles (ca. 4 to $5 \mathrm{~m}$ ) as well as the scaling laws of manufacturing cost. In order to overcome this difficulty, break-through technologies such as a Separated Spacecraft Interferometer (SSI) are being studied for NASA's Terrestrial Planet Finder (TPF) mission. Other benefits to using such a system include array reconfigurability and upgradability. Another formation flight concept that has been considered for a SSI system is the use of tether. To image a target, measurements must be made in all directions orthogonal to the line-of-sight of the array. The balance between using a Structurally Connected Interferometer (SCI), which allows for very limited baseline changes, and a SSI system where the usage of propellant can be prohibitively expensive, seems to be using a tethered system. Such a system is currently being considered for NASA's Sub-millimeter Probe of the Evolution of Cosmic Structure (SPECS) mission. ${ }^{1}$ One mission concept is to use a Tethered Spacecraft Interferometer (TSI) system to maneuver the subapertures out to separations of a kilometer, thereby achieving very high resolution. Since power, maneuvering loads and data can be supported by the tether, these typical spacecraft functions are not required on the maneuvering vehicles. This reduces replication of sub-systems across the various sub-apertures and eliminates the need for propellant. Furthermore, the mass per unit length of the tether is much smaller than that of a deployed truss making it much more mass-efficient, particularly for long baselines. However, all of these benefits are lost if the control needed to achieve the precision required by the array proves to be too complex. It is expected that vibratory motion, consisting of compound pendulum mode of the satellite bus and tether violin modes, will be observed during operations of a TSI. Highly maneuverable spacecraft are particularly problematic since beam control in the optics system will need to be maintained to the requisite precision while thrusters fire, tethers vibrate, and reaction wheels change momentum. These introduce harsh disturbances that necessitate the coupling of attitude and optical control. The tethered SPHERES project aims at addressing all the issues associated

Further author information: (Send correspondence to sjchung@mit.edu.)

S.-J. Chung: Doctoral Student, Department of Aeronautics and Astronautics, MIT, E-mail: sjchung@mit.edu

E. M. Kong: Research Scientist, MIT Space Systems Lab, E-mail: mckong@mit.edu

D. W. Miller: Associate Professor, Department of Aeronautics and Astronautics, MIT, E-mail:millerd@mit.edu

UV/Optical/IR Space Telescopes: Innovative Technologies and Concepts II, edited by Howard A. MacEwen, Proceedings of SPIE Vol. 5899 (SPIE, Bellingham, WA, 2005) · 0277-786X/05/\$15 · doi: 10.1117/12.614643

Proc. of SPIE 58990L-1 
with tethered formation flight. Moreover, the lessons learned from the SPHERES experiments will facilitate the development of sophisticated tether and attitude controllers for the future tethered spacecraft missions.

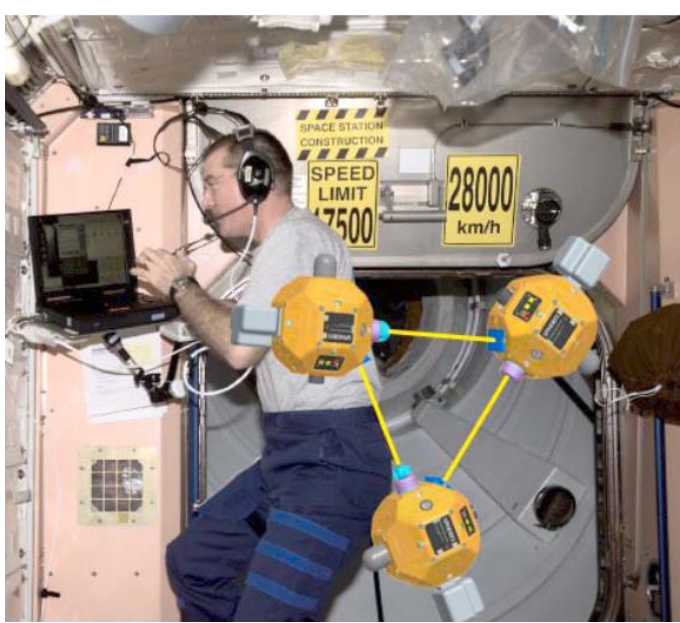

Figure 1. Artist's concept of tethered formation flying SPHERES in ISS cell

\subsection{Objective}

The objective of this project is to demonstrate the feasibility of controlling array formation (array re-sizing, array spin-up, array deployment) without thrusters for the future space interferometer missions. No propellant thrusters mean unlimited electric power available via the solar energy for the actuators of formation flight (reaction wheels and tether motor). It is also beneficial to the science since there will be no optics contamination from thruster plumes. Another goal is to develop sophisticated decentralized control and estimation algorithms for the tethered system reducing the need for satellite communications. The relative metrology system supporting this decentralized estimation and control should be also developed and validated experimentally.

\subsection{Previous Work}

The Synchronized Position Hold Engage and Reorient Experimental Satellites (SPHERES) testbed will be the first satellite testbed fully exploiting the dynamics of tethered satellites under various formation flight configurations. It is also a fully $3-\mathrm{D}$ operational satellite with sophisticated sensors and actuators as a mode-representative of the real satellites in orbit. A notable previous work on experiments of two tethered satellites is the Ph.D thesis by Motley. ${ }^{3}$ A recent paper also describes the tethered testbed. ${ }^{4}$ There is a lot of literature on the dynamics of tether in space. Some recent papers discuss the elasticity and vibration of the tethers. ${ }^{5,6}$ Compared to the early literature focused on the two-body dynamics for the tether retrieval and momentum exchange purposes, more recent work investigates the dynamics of a three-body inline configuration ${ }^{8}$ and a triangular configuration. ${ }^{9}$ This paper studies both configurations for the SPECS mission as detailed in Sec 3.2. Development of nonlinear controller was performed for the SPECS mission by Mischa, ${ }^{7}$ but this model does not fully account for the pendulum libration mode and lacks experimental validation.

\section{DEVELOPMENT OF SPHERES HARDWARE}

The MIT SPHERES testbed provides a vehicle to demonstrate and validate formation flight and docking technologies for use in missions such as TPF and Orbital Express. Among the technologies that are actively under study by SPHERES are space interferometry, cluster reconfiguration, and mission re-supply. Many of these techniques can only be tested in simulation or with expensive and risky flight projects. Currently, there are no on-orbit resources suitable for the validation of general simulation results, and most space missions do not push the limits of performance due to the high risk associated with flying unproven algorithms. The SPHERES testbed is an inexpensive and risk-tolerant laboratory for the validation of distributed spacecraft control, estimation, and autonomy algorithms. It fills the gap between the flexibility, risk-tolerance, and uncertainty of simulation-based research and the inflexibility, expense, and credibility expected from future space flight missions. The SPHERES testbed was developed as part of the ongoing research initiatives of the MIT Space Systems Laboratory (MITSSL) that utilize the space environment provided by the space shuttle and International Space Station (ISS) to validate dynamics and control algorithms.

\subsection{Tethered SPHERES Overview}


The basic setup of the SPHERES testbed consists of a number of autonomous free-flyers satellites, a laptop computer that serves as a ground station, and small beacons that form the Position and Attitude Determination System (PADS). SPHERES has three main operational environments: simulation, flat floor facility (at MIT and NASA Marshall Space Flight Center), and 3D environment (inside the ISS and NASA's KC-135 flight). The simulation allows the operation of up to three satellite models in any standard PC running Windows operating system. The configuration at the SSL facility consists of multiple operational flight-qualified SPHERES satellites, a metrology setup optimized for 2D operations, and a metrology setup designed for 3D operations. The final ISS configuration will consist of three tethered satellites and a 3D metrology setup (See Fig. 1). Both MIT-SSL and ISS setups use a laptop computer to represent a ground control station; through the laptop, the user runs tests and collects telemetry data for future analysis. The laptop computer utilizes a custom communications device to control the satellites and store all the data. Different interfaces were developed for the ISS and ground operations; the ground interface minimizes the time between tests, while the ISS operations clearly steps through the operation procedures to ensure correct tests are being implemented.

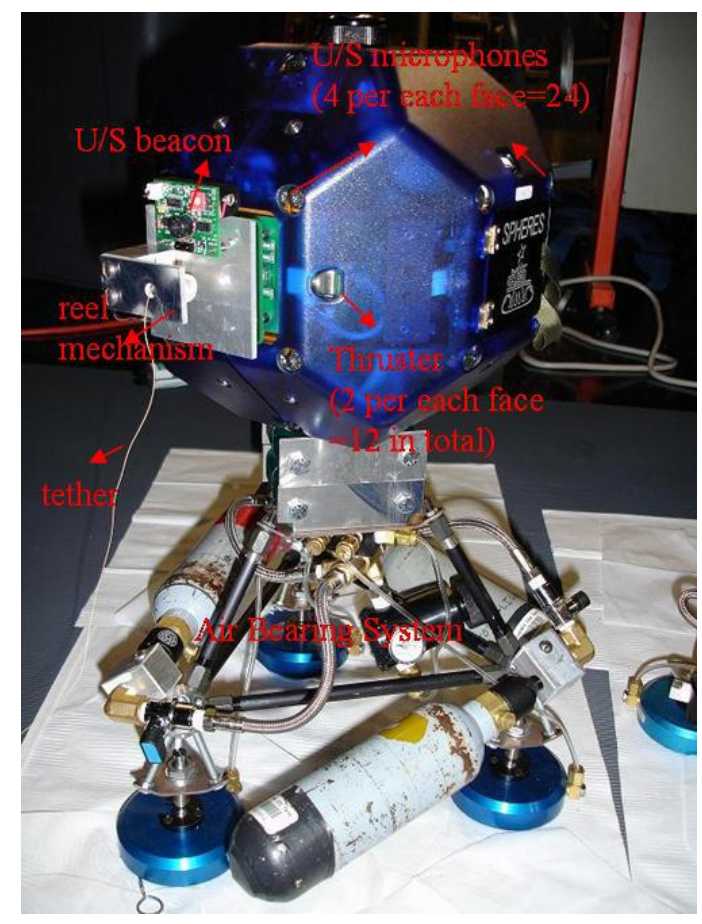

\subsection{SPHERES Satellites}

The individual self-contained satellites (Fig. 2) have the ability to maneuver in up to six degrees of freedom*, to communicate with

Figure 2. SPHERES satellite with air-bearing carriage each other and with the laptop control station, and to identify their position with respect to each other and to the experiment reference frame. The diameter of a single SPHERES is $0.25 \mathrm{~m}$, and the mass is $4.0 \mathrm{~kg}$. The satellites are propelled by a cold-gas thruster system which uses carbon dioxide as propellant. The CO2 propellant is stored in liquid form at 860 psig; a regulator reduces the pressure to 35 psig. Twelve thrusters are positioned to provide controllability in all six degrees of freedom, enabling both torque and translation control. Each thruster assembly consists of a solenoid-actuated micro-valve with machined nozzles. Depending upon the tests of interest, a single tank provides approximately 30 minutes of active operation. After each test session, a tank can be left in the system partially full, for use at a later time, or be replaced with a new tank. The PADS provides metrology information to the satellites in real-time. Since no global metrology system like GPS is actually available in deep space missions, the tethered SPHERES system utilizes a relative metrology system using four ultrasound receivers on the line-of-sight face and the on-board beacon of the adjacent SPHERES (see Fig. 2). The relative metrology system is a pseudo-GPS ranging system that uses ultrasonic time-of-flight measurements from the target on-board beacon to the ultrasonic microphones distributed on the surface of each satellite. These timeof-flight measurements are converted to ranges and are then used to derive position and attitude with respect to the reference frame using a series of Extended Kalman Filters (EKFs). A detailed description on the relative metrology system will follow in section 5. A Texas Instruments C6701 Digital Signal Processor provides the computational power. The ability of the C6701 to provide up to 1.0 GFLOPS provides significant processing power to prevent being the limiting factor in the performance of the system. A FLASH memory size of 224 KB allows software re-configuration of the full operating system, ensuring that multiple investigators are supported while the system is in the ISS. The power system utilized onboard the ISS consists of packs of AA alkaline batteries while NiMH rechargeable packs are used on the ground facility. The packs provide each satellite with approximately two hours of operation; once a pack is consumed, it can be easily replaced. Each SPHERES satellite uses two separate frequency communications channels operating at $57.6 \mathrm{kbps}$ each. One channel is used for satellite-to-satellite communications; the other channel enables satellite-to-laptop communications. Both

\footnotetext{
* or three degrees of freedom on the flat floor
} 
channels are bi-directional; however, the communication hardware is half-duplex, meaning that only one unit can transmit at a time.

\subsection{Modification for Tethered Formation}

The tether package can be divided into three major components: the tether deployment and retraction mechanism with tether tension sensors; latch plate using velcro; and momentum wheel package. Prior to implementing the reaction wheels, the torque generated by diagonal thrusters is utilized in the experiments presented in this paper. The reel in/out mechanism is used to control the tethers during extension and contraction. It allows the tether to smoothly and orderly maintain a tension between satellites. The tether from the deployment mechanism is connected to the latch plate on the other satellite. The momentum wheel package, which consists of 3 wheels, will be located on the opposite side of the tether mechanism to counter-balance the weight introduced by the tether mechanism. Note that both the tether deployment mechanism and momentum wheel assembly are controlled through the satellite's expansion port and are designed to be modular where one design fits all three satellites. The relative metrology system provides measure of the separation distance for feedback control. The completion of this work will result in a flight rated tether mechanism ready for integration onboard the Shuttle or ISS. With this hardware, dynamics and control work are studied and demonstrated on a 2-D flat floor facility prior to testing in a more realistic 3-D environment, onboard the ISS.

\section{NONLINEAR DYNAMICS MODELING}

\subsection{Assumptions}

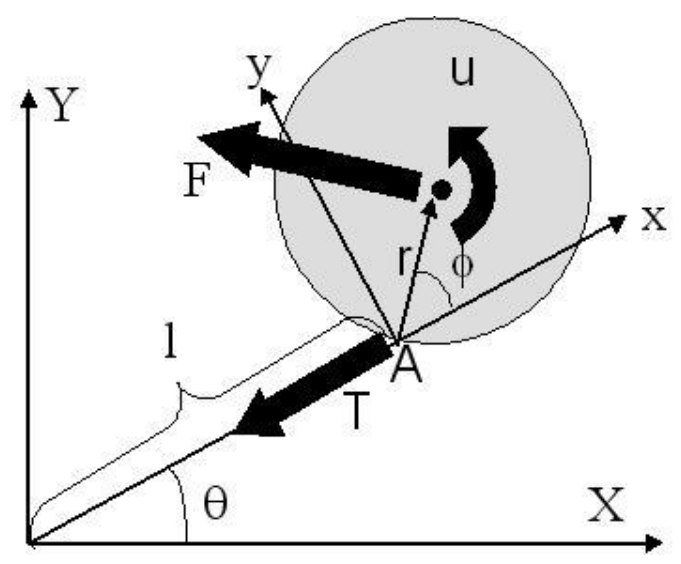

For the sake of convenience, the dynamics relevant only to the 2D flat floor test is formulated here before the full $3 \mathrm{D}$ equations accounting for out-of-plane motions are investigated. Fig. 3 shows a single SPHERES revolving about the center of the fixed inertial axes $\mathrm{X}-\mathrm{Y}$. The rotating axes, $\mathrm{x}-\mathrm{y}$ are chosen such that the $\mathrm{y}$ direction always coincides with the direction of increasing $\theta$ while the $\mathrm{x}$ direction is aligned with the tether. The SPHERES is also exhibiting a compound pendulum rotation with respect to $\mathrm{A}$. The $\mathrm{CM}$ of the SPHERES is Point B. The force and torque exerted on the CM (Point B) are drawn in black. $T$ is the tension force of the tether, $F_{x}$ and $F_{y}$ are the x-y directional forces due to thruster firing, and $u$ is the torque exerted on the CM (Center of Mass)-point B, e.g torque by Reaction Wheel Assembly (RWA).

The following assumptions are made regarding the tether wire. The tether is massless and inextensible, however it is planned to account for the effect of the elasticity of the tether in the near

Figure 3. Free-body diagram of a revolving tether problem future. Additionally, the array is assumed to always rotate at a certain angular rate so the tether is taut and straight at all times. A non-zero angular rotation is a realistic assumption since the interferometer will attempt to fill a full $\mathrm{u}-\mathrm{v}$ coverage by rotation. However, it was observed from the experiments that the assumption of tautness is no longer valid when the satellites bounce off the tether by high-impedance actuation. We plan to mitigate this effect by adding some flexibility to the tether (e.g. spring). We also assume a constant reel-in or reel-out speed.

\subsection{Derivation of Nonlinear Equations}

In order to reduce the complexity of trigonometric functions (sine and cosine), the velocity and acceleration will be expressed in the small x-y rotating frame and the corresponding unit directional vectors are $e_{x}$ and $e_{y}$.

$$
v_{B}=[i-r \sin \phi(\dot{\theta}+\dot{\phi})] e_{x}+[r \cos \phi(\dot{\theta}+\dot{\phi})+l \dot{\theta}] e_{y}
$$


The relationship between the absolute and relative acceleration is obtained by differentiating the relative velocity equation for the fixed tether length to get

$$
\begin{array}{r}
a_{B}=e_{x}\left[-l \dot{\theta}^{2}-r \sin \phi \ddot{\theta}-r \cos \phi \dot{\theta}^{2}-2 r \cos \phi \dot{\theta} \dot{\phi}-r \ddot{\phi} \sin \phi-r \dot{\phi}^{2} \cos \phi\right]+ \\
e_{y}\left[(l+r \cos \phi) \ddot{\theta}-r \sin \phi \dot{\theta}^{2}-2 r \sin \phi \dot{\theta} \dot{\phi}+r \ddot{\phi} \cos \phi-r \dot{\phi}^{2} \sin \phi\right]
\end{array}
$$

The equations of the tethered system is derived using Eq. (2):

$$
\begin{array}{r}
\sum F_{e_{x}}=-F_{x}-T=-F \sin \phi-T=m a_{x} \\
\sum F_{e_{y}}=F_{y}=F \cos \phi=m a_{y} \\
\sum M_{G}=-T r \sin \phi+u=I_{G}(\ddot{\theta}+\ddot{\phi})
\end{array}
$$

where $M_{G}$ is the moment around $\mathrm{CM}$ (point B) and $I_{G}$ denotes the moment of inertia around CM. $a_{x}$ and $a_{y}$ are the x,y acceleration components of Eq.(2) respectively.

$T$ can be eliminated and the following differential equation is obtained,

$$
[M(\phi)]\left(\begin{array}{c}
\ddot{\theta} \\
\ddot{\phi}
\end{array}\right)+[C(\phi, \dot{\theta}, \dot{\phi})]=\left(\begin{array}{c}
F \cos \phi \\
F r+u
\end{array}\right)
$$

where $[M(\phi)]=\left[\begin{array}{cc}m l+m r \cos \phi & m r \cos \phi \\ I_{G}+m r^{2}+m r l \cos \phi & I_{G}+m r^{2}\end{array}\right]$ and

$[C(\phi, \dot{\theta}, \dot{\phi})]=\left[\begin{array}{c}-2 m r \sin \phi \dot{\theta} \dot{\phi}-m r \sin \phi \dot{\theta}^{2}-m r \sin \phi \dot{\phi}^{2} \\ m r l \sin \phi \dot{\theta}^{2}\end{array}\right]$

When the tether motor reels in or out at a constant speed $(i=$ constant), the force term in Eq. (4) becomes

$$
\left(\begin{array}{c}
F_{y} \\
u
\end{array}\right) \Rightarrow\left(\begin{array}{c}
F_{y}-2 m i \dot{\theta} \\
u
\end{array}\right)
$$

The equations of the motion can be also derived by exploiting the techniques developed for multi-link robot kinematics or Lagrange's equation. Those equations are simplified assuming the mass of the first link is zero (therefore, the inertia of moment is zero as well). Then, the governing equation of motion becomes

$$
[M(\phi)]\left(\begin{array}{c}
\ddot{\theta} \\
\ddot{\phi}
\end{array}\right)+[C(\phi, \dot{\theta}, \dot{\phi})]=\left(\begin{array}{c}
\tau_{1} \\
\tau_{2}
\end{array}\right)
$$

where $[M(\phi)]=\left[\begin{array}{cc}I_{G}+m r^{2}+m l^{2}+2 m r l \cos \phi & I_{G}+m r^{2}+m r l \cos \phi \\ I_{G}+m r^{2}+m r l \cos \phi & I_{G}+m r^{2}\end{array}\right]$ and $[C(\phi, \dot{\theta}, \dot{\phi})]=\left[\begin{array}{c}-2 m r l \sin \phi \dot{\theta} \dot{\phi}-m r l \sin \phi \dot{\phi}^{2} \\ m r l \sin \phi \dot{\theta}^{2}\end{array}\right]$

Compared to Eq.(4), Eq.(6) has more simpler form of $C(\phi, \dot{\theta}, \dot{\phi})$ function and the inertia matrix, $M$ is now symmetric. The torque $\tau_{1}$ and $\tau_{2}$ are now applied to the joints $\mathrm{O}$ (origin of $\mathrm{X}-\mathrm{Y}$ ) and $\mathrm{A}$ (origin of $\mathrm{x}-\mathrm{y}$ ) respectively.

It is shown Eq.(4) and Eq.(6) are essentially the same equations since 1st row times $l$ plus 2nd row of Eq.4 results in the first eqn of Eq.6.

So the external force(or torque) terms can be matched like the following:

$$
\left(\begin{array}{c}
\tau_{1} \\
\tau_{2}
\end{array}\right)=\left[\begin{array}{cc}
r+l \cos \phi & 1 \\
r & 1
\end{array}\right]\left(\begin{array}{c}
F \\
u
\end{array}\right)
$$




\subsection{Multiple Vehicle Dynamics}

The dynamics of two satellites given by Lagrangian equation is derived. The assumption of all identical spacecraft in size, mass and inertia property, allows us to develop the following equation of the motion:

$$
\begin{aligned}
& {[M(\phi)]\left(\begin{array}{c}
\ddot{\theta} \\
\ddot{\phi_{1}} \\
\ddot{\phi_{2}}
\end{array}\right)+\left[C\left(\phi_{1}, \phi_{2}, \dot{\theta}, \dot{\phi}_{1}, \dot{\phi}_{2}\right)\right]=\left(\begin{array}{c}
u_{1}+u_{2} \\
u_{1} \\
u_{2}
\end{array}\right)} \\
& \text { where }[M(\phi)]=\left[\begin{array}{ccc}
2 I_{G}+2 m r^{2}+2 m l^{2}+2 m r l \cos \phi_{1}+2 m r l \cos \phi_{2} & I_{G}+m r^{2}+m r l \cos \phi_{1} & I_{G}+m r^{2}+m r l \cos \phi_{2} \\
I_{G}+m r^{2}+m r l \cos \phi_{1} & I_{G}+m r^{2} & 0 \\
I_{G}+m r^{2}+m r l \cos \phi_{2} & 0 & I_{G}+m r^{2}
\end{array}\right] \\
& {\left[C\left(\phi_{1}, \phi_{2}, \dot{\theta}, \dot{\phi_{1}}, \dot{\phi_{2}}\right)\right]=\left[\begin{array}{c}
-2 m r l \dot{\theta}\left(\sin \phi_{1} \dot{\phi}_{1}+\sin \phi_{2} \dot{\phi}_{2}\right)-m r l \sin \phi_{1}{\dot{\phi_{1}}}^{2}-m r l \sin \phi_{2}{\dot{\phi_{2}}}^{2} \\
m r l \sin \phi_{1} \dot{\theta}^{2} \\
m r l \sin \phi_{2} \dot{\theta}^{2}
\end{array}\right]}
\end{aligned}
$$

The array angular rate $\dot{\theta}$ is assumed to be the same for both

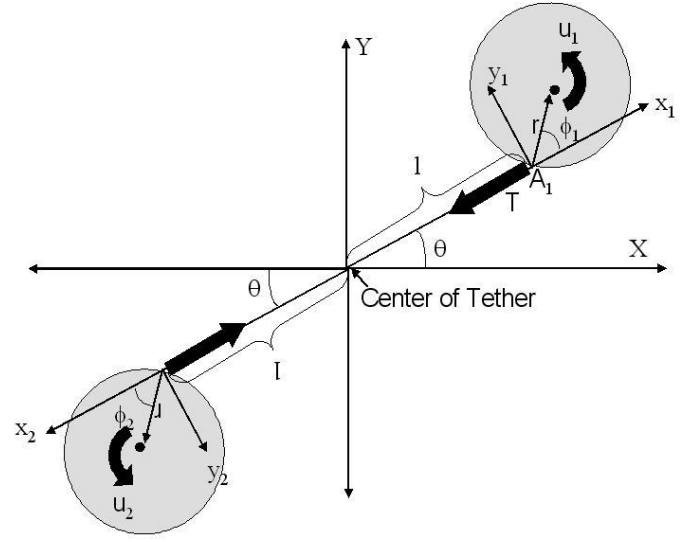

Figure 4. Free-body diagram of tethered two SPHERES satellites. This is especially true when the tether is in tension. Furthermore, the mass and the inertia properties are assumed to be roughly the same. If the initial condition of the pendulum mode, $\phi$ and $\dot{\phi}$ of both satellites are the same, then we can decouple the dynamics of the first SPHERES from the other resulting in the same equation as Eq. (6). This means a decentralized controller can be exploited to control two tethered satellites without the need for communication of each other's states information. This will significantly simplify both control algorithm and satellite hardware with less complication. Basically the fixed center of the rotation for a single tethered system will be replaced with the center of mass (CM) of the two SPHERES (See Fig. 4).

Two configurations for three tethered spacecraft are being considered for the SPECS mission as shown in Fig. 5. The performance of two configurations are yet to be compared in the near future. Motivated by successful decoupling for the two spacecraft case, three imaginary pseudo-tethers connecting each satellite to the Center of Mass (CM) of the array are assumed to be present replacing the three actual tether lines (see the dashed lines in Fig. 5). When the tethers are taut and straight in a rotating array, a small $\phi$ (angle of pendulum mode) is approximated as a perturbed angle that the SPHERES makes with respect to the corresponding pseudo-tether. Then, the formulation of the dynamics of each SPHERES results in the single tether case Eq.(4) and (6). The length of the pseudo-tether is the actual tether length divided by $\sqrt{3}$. This psuedo-tether technique is yet to be compared with the actual dynamics obtained by Lagrange's equation. However, the controller based on this technique performed well in the experiments. More in-depth analysis on the decentralized controller can be found at the reference. ${ }^{10}$

\section{LINEARIZATION AND LINEAR CONTROLLER}

\subsection{Linearization and Natural Frequency}

We will linearize Eq.(6) about $\dot{\theta}=\omega$, and $\dot{\phi}, \phi=0$. Each term can be linearized as the following: $m r l \sin \phi \dot{\theta}^{2} \approx$ $m r l \omega^{2} \phi, m r l \sin \phi \dot{\phi}^{2} \approx 0, m r l \sin \phi \dot{\theta} \dot{\phi} \approx 0, \cos \phi \approx 1$. The linearized equation of the motion is presented:

$$
\left[\begin{array}{cc}
I_{G}+m(r+l)^{2} & I_{G}+m r(r+l) \\
I_{G}+m r(r+l) & I_{G}+m r^{2}
\end{array}\right]\left(\begin{array}{c}
\ddot{\theta} \\
\ddot{\phi}
\end{array}\right)+\left[\begin{array}{cc}
0 & 0 \\
0 & m r l \omega^{2}
\end{array}\right]\left(\begin{array}{c}
\theta \\
\phi
\end{array}\right)=\left(\begin{array}{l}
\tau_{1} \\
\tau_{2}
\end{array}\right)
$$




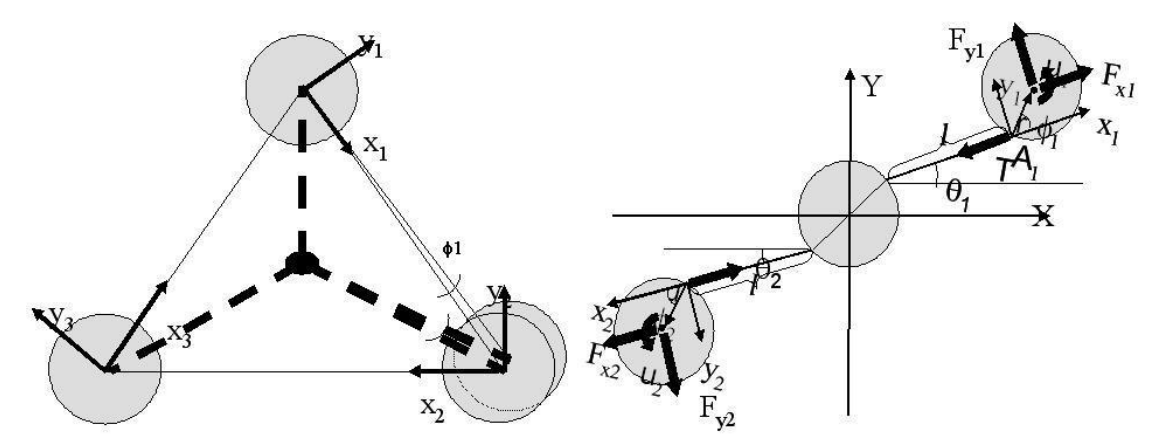

Figure 5. Two possible configurations for three tethered satellites for SPECS

Similarly, we can linearize Eq.(4) and transformation between the two system equations is easily performed using Eq.(7).

A nice property about this linearized equation (9) is that we can decouple the equation of $\phi$ from that of $\theta$. The first equation is merely the dynamics of a rigid body mode of $\theta$ (rotation about the center of X-Y).

Since the inertia matrix is invertible, we multiply both sides of Eq.(9) by the inverse of $M$ matrix:

$$
\left(\begin{array}{c}
\ddot{\theta} \\
\ddot{\phi}
\end{array}\right)+\left[\begin{array}{cc}
0 & -\frac{r \omega^{2}\left(I_{G}+m r(r+l)\right)}{l I_{G}} \\
0 & \frac{r \omega^{2}\left(I_{G}+m(r+l)^{2}\right)}{l I_{G}}
\end{array}\right]\left(\begin{array}{l}
\theta \\
\phi
\end{array}\right)=\frac{1}{I_{G} m l^{2}}\left[\begin{array}{cc}
I_{G}+m r^{2} & -I_{G}-m r(r+l) \\
-I_{G}-m r(r+l) & I_{G}+m(l+r)^{2}
\end{array}\right]\left(\begin{array}{c}
\tau_{1} \\
\tau_{2}
\end{array}\right)
$$

The second-order nonlinear equation of motion of $\phi$ from the second line of (10) becomes:

$$
\ddot{\phi}+\omega_{\phi}^{2} \phi=\frac{-I_{G}-m r(r+l) \tau_{1}+I_{G}+m(l+r)^{2} \tau_{2}}{I_{G} m l^{2}}
$$

where $f$ is the frequency of the pendular libration mode:

$$
\omega_{\phi}=\sqrt{\frac{I_{G} r \omega^{2}+m r \omega^{2}(r+l)^{2}}{l I_{G}}}[\mathrm{rad} / \mathrm{s}]
$$

If we want to express the external torque terms of Eq.(10) in terms of $F_{y}$ (thruster firing to the y direction) and $u$ (torque exterted on the CM of the SPHERES by e.g. RWA) We linearize the relationship of Eq.(7):

$$
\left(\begin{array}{l}
\tau_{1} \\
\tau_{2}
\end{array}\right)=\left[\begin{array}{cc}
l+r & 1 \\
r & 1
\end{array}\right]\left(\begin{array}{c}
F_{y} \\
u
\end{array}\right)
$$

Then Eq.(10) becomes

$$
\left(\begin{array}{c}
\ddot{\theta} \\
\ddot{\phi}
\end{array}\right)+\left[\begin{array}{cc}
0 & -\frac{r \omega^{2}\left(I_{G}+m r(r+l)\right)}{l I_{G}} \\
0 & \frac{r \omega^{2}\left(I_{G}+m(r+l)^{2}\right)}{l I_{G}}
\end{array}\right]\left(\begin{array}{l}
\theta \\
\phi
\end{array}\right)=\left[\begin{array}{cc}
\frac{1}{m l} & -\frac{r}{I_{G} l} \\
-\frac{1}{m l} & \frac{r+l}{I_{G} l}
\end{array}\right]\left(\begin{array}{c}
F_{y} \\
u
\end{array}\right)
$$

The actual raw gyro data from a single tethered SPHERES exhibit a high frequency oscillation (the pendulum mode) and the DC component (a rigid body mode of a certain rotational rate). The frequency obtained by Eq. (12) correctly predicted the actual frequency obtained from the gyro data with a less than 5 percent error. 


\subsection{Controllability}

The Linear Time Invariant (LTI) system is presented from Eq. (14) as the following.

$$
\frac{d}{d t}\left(\begin{array}{c}
\theta \\
\phi \\
\dot{\theta} \\
\dot{\phi}
\end{array}\right)=\left[\begin{array}{cccc}
0 & 0 & 1 & 0 \\
0 & 0 & 0 & 1 \\
0 & \frac{r \omega^{2}\left(I_{G}+m r(r+l)\right)}{l I_{G}} & 0 & 0 \\
0 & -\frac{r \omega^{2}\left(I_{G}+m(r+l)^{2}\right)}{l I_{G}} & 0 & 0
\end{array}\right]\left(\begin{array}{c}
\theta \\
\phi \\
\dot{\theta} \\
\dot{\phi}
\end{array}\right)+\left[\begin{array}{cc}
0 & 0 \\
0 & 0 \\
\frac{1}{m l} & -\frac{r}{I_{I} l} \\
-\frac{1}{m l} & \frac{r+l}{I_{G} l}
\end{array}\right]\left(\begin{array}{c}
F_{y} \\
u
\end{array}\right)
$$

where the first matrix of the righthand side is the $4 \times 4$ system matrix $A$ and the second is the $4 \times 2$ input matrix $B$. Note that $\theta$ is easily eliminated by removing the first column and the first of row of $A$ matrix, thereby reducing the dimension to three.

Let's check if the system is really controllable around nominal points by calculating controllability matrix.

$$
\left[\begin{array}{llll}
B & A B & A^{2} B & A^{3} B
\end{array}\right]=\left[\begin{array}{cccc}
0 & 0 & \frac{1}{m l} & -\frac{r}{I_{G} l} \\
0 & 0 & -\frac{1}{m l} & \frac{r+l}{I_{G} l} \\
\frac{1}{m l} & -\frac{r}{I_{G} l} & 0 & 0 \\
-\frac{1}{m l} & \frac{r+l}{I_{G} l} & 0 & 0
\end{array}\right]
$$

Where the calculation of $A^{2} B$ and $A^{3} B$ is omitted since the first two matrices result in the full rank $(n=4)$ and the rest of them are redundant (dependent). So the system is fully controllable with $u$ (Control torque on CM by RWA or coupled thruster firing) and $F_{y}$ (linear thruster firing).

In fact, all the states $(\theta, \phi, \dot{\theta}, \dot{\phi})$ are controllable only by the torque generating actuator $u$ (e.g. RWA). The controllability matrix using the second column of $B$ matrix in eqn(15) becomes:

$$
\left[\begin{array}{llll}
B_{2} & A B_{2} & A^{2} B_{2} & A^{3} B_{2}
\end{array}\right]=\left[\begin{array}{cccc}
0 & -\frac{r}{I_{G} l} & 0 & \frac{r(r+l) \omega^{2}\left(I_{G}+m r(r+l)\right)}{l^{2} I_{G}{ }^{2}} \\
0 & \frac{r+l}{I_{G} l} & 0 & -\frac{r(r+l) \omega^{2}\left(I_{G}+m(r+l)^{2}\right)}{l^{2} I_{G}{ }^{2}} \\
-\frac{r}{I_{G} l} & 0 & \frac{r(r+l) \omega^{2}\left(I_{G}+m r(r+l)\right)}{l^{2} I_{G}{ }^{2}} & 0 \\
\frac{r+l}{I_{G} l} & 0 & -\frac{r(r+l) \omega^{2}\left(I_{G}+m(r+l)^{2}\right)}{l^{2} I_{G}{ }^{2}} & 0
\end{array}\right]
$$

This is a full rank $(n=4)$ matrix. Its implication to the future tethered systems is enormous: the tethered satellite systems will be able to spin up and re-size the array on the plane of the array without the use of propellant consumable when operating at nominal points (slowly varying rotational rate with small pendulum mode). This linear controller implemented in this paper takes advantage of this observation. The restricting condition is that the system is only controllable around the nominal states.

\subsection{Effect of Varying Tether Length}

In the previous sections, the length of the tether is assumed to be invariant $(i=0)$. The dynamics of varying tether length with a constant motor reel-in/out speed $(i=$ constant), is investigated in this section.

Using Eq. (5), we modify the linearized equation (14) as the following:

$$
\left(\begin{array}{c}
\ddot{\theta} \\
\ddot{\phi}
\end{array}\right)+\left[\begin{array}{cc}
2 \frac{i}{l} & 0 \\
-2 \frac{i}{l} & 0
\end{array}\right]\left(\begin{array}{c}
\dot{\theta} \\
\dot{\phi}
\end{array}\right)+\left[\begin{array}{cc}
0 & -\frac{r \omega^{2}\left(I_{G}+m r(r+l)\right)}{l I_{G}} \\
0 & \frac{r \omega^{2}\left(I_{G}+m(r+l)^{2}\right)}{l I_{G}}
\end{array}\right]\left(\begin{array}{c}
\theta \\
\phi
\end{array}\right)=\left[\begin{array}{cc}
\frac{1}{m l} & -\frac{r}{I_{G} l} \\
-\frac{1}{m l} & \frac{r+l}{I_{G} l}
\end{array}\right]\left(\begin{array}{c}
F_{y} \\
u
\end{array}\right)
$$


Similarly, the LTI equation (15) is modified into:

$$
\frac{d}{d t}\left(\begin{array}{c}
\theta \\
\phi \\
\dot{\theta} \\
\dot{\phi}
\end{array}\right)=\left[\begin{array}{cccc}
0 & 0 & 1 & 0 \\
0 & 0 & 0 & 1 \\
0 & \frac{r \omega^{2}\left(I_{G}+m r(r+l)\right)}{l I_{G}} & -2 \frac{i}{l} & 0 \\
0 & -\frac{r \omega^{2}\left(I_{G}+m(r+l)^{2}\right)}{l I_{G}} & 2 \frac{i}{l} & 0
\end{array}\right]\left(\begin{array}{c}
\theta \\
\phi \\
\dot{\theta} \\
\dot{\phi}
\end{array}\right)+\left[\begin{array}{cc}
0 & 0 \\
0 & 0 \\
\frac{1}{m l} & -\frac{r}{I_{G} l} \\
-\frac{1}{m l} & \frac{r+l}{I_{G} l}
\end{array}\right]\left(\begin{array}{c}
F_{y} \\
u
\end{array}\right)
$$

The nonlinear equations can be easily modified in the same fashion.

Instead of showing an analytic solutions of the eigenvalues of Eq. (19), a real-imaginary axes plot is presented as shown in Fig. 6. It is noted that the system is stable when the motor reels out. In other words, a positive reel-out speed resulted in damping of the pendulum motion of $\phi$. However, we will see UNSTABLE motion of $\phi$ for a negative reel-in speed. In conclusion, we need a stabilizing controller for the system with decreasing tether length.

\section{SENSOR SUITE AND ESTIMATION}

The control techniques introduced in the subsequent section are based upon the supposition that all the states are strictly available for feedback. In this section, a description on the relative metrology sensor suite and estimator design to estimate the system states $(\phi, \dot{\theta}, \dot{\phi})$ is elaborated.

The role of the relative estimator is to provide autonomous state estimates (tether length, and all the states including the bearing angle $\phi$ ) independently of adjacent satellites. It is desir-

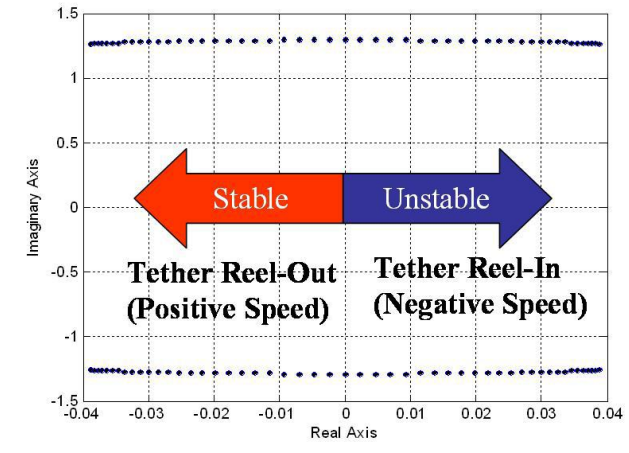

Figure 6. Complex poles of a tethered system with the tether speed ranging from $-0.5 \mathrm{~m} / \mathrm{s}$ to $+0.5 \mathrm{~m} / \mathrm{s}$ able to maintain the independency to avoid any possible communication between satellites. Its schematic is illustrated in Fig. 7. Each SPHERES satellite is equipped with a beacon located on its docking face. An additional on-board beacon is also mounted on the expansion port face where the tether mechanism package is attached (See figure 2). Those 24 ultrasound receivers combined with a gyroscope simulate a GPS - IMU sensor suite providing all the necessary system states.

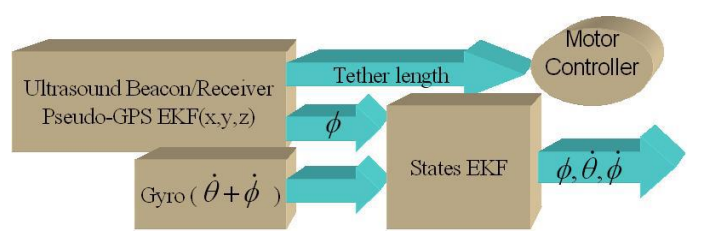

Figure 7. Schematic of the SPHERES relative metrology system
This pseudo-GPS system estimates the $\mathrm{x}, \mathrm{y}, \mathrm{z}$ coordinates of the incoming beacon in the body frame of the SPHERES as depicted in figure 8. The measurement process is similar to using the time gap between seeing the lightening and hearing the thunder clap to calculate how far you are away from a thunderstorm. The method by which the on-board beacons are used to compute relative position and attitude is as following. ${ }^{2}$ When the leader SPHERES requests a next state update, it emits an infrared ping which is received by all the surrounding SPHERES and the

external on-board beacons. All the SPHERES then start to listen for an ultrasound ping through their 24 ultrasound receivers located all around them. The beacons emit an ultrasound ping one after the other (20 milliseconds apart) to avoid the interference. The pings are then received by each SPHERES and an array of time-of-flight data is sent to the computer. Since the range measurement is nonlinear, the EKF is employed to estimate the range in terms of $\mathrm{x}, \mathrm{y}, \mathrm{z}$, coordinate values. Once the pseudo-GPS system estimates $\mathrm{x}, \mathrm{y}, \mathrm{z}$ coordinates of the target beacon, the tether length $l$ and the bearing pendulum mode angle $\phi$ are computed from the geometry shown in figure 8:

$$
l=\sqrt{(x-r)^{2}+y^{2}+z^{2}}
$$




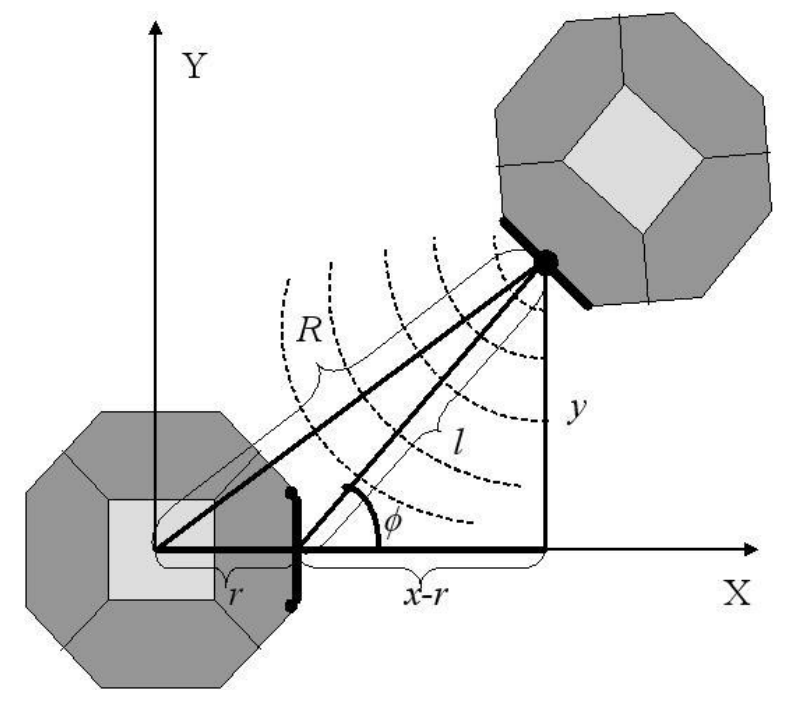

Figure 8. Pendulum angle determination using relative metrology system

$$
\phi=\arcsin (y / l)
$$

The tether length is used in realtime for the tether-motor control whereas the direct $\phi$ measurement is used as complementary filtering with the high-frequency gyro measurement (see figure 7). The complementary EKF makes use of the nonlinear equation developed in section 3.2 in order to estimate $\phi, \dot{\theta}$, and $\dot{\phi}$.

\section{LINEAR CONTROL AND EXPERIMENTAL RESULTS}

Section 6 and 7 include the experiment results obtained at the NASA Marshall Space Flight Center, December 2004. More experiments will be conducted in the first half of 2005. Now we can easily construct a linear controller with the LTI system given by Eq.(15). We intentionally eliminate the state $\theta$ from Eq.(15) since it is merely a rigid body mode. So all the states are available by the estimator in the previous section. We can design a Linear Quadratic Regulator (LQR) controller, which minimizes the following cost function, which specifically addresses the issue of achieving a balance between good system response and the control effort required. Since the A matrix is a function of $\dot{\theta}$ and the tether length $l$, the optimal LQR gains are calculated over various angular rate and tether length. Since we are going to increase the tether length after achieving a certain angular rate, a gain-scheduled LQR gain is a continuous function of $\dot{\theta}$ over discrete tether length. Some of the curvefitted functions resulted in a nonlinear quadratic function of $\dot{\theta}$. This gain-scheduled LQR controller has been successfully implemented in the tethered SPHERES testbed. Figure 9 shows the states background telemetry information directly recorded via the communication link from the satellite. The green line indicates the array angular rate $(\dot{\theta})$;blue is $\phi$;red is $\dot{\phi}$;light blue is gyro data. The second row of the figure represents the actuator output (the torque has a saturation limit at $0.012 \mathrm{Nm}$ ). The LQR controller tried to spin up a satellites into 0.3 $\mathrm{rad} / \mathrm{s}$ from some arbitrary maneuvers. This control utilized only torque actuators to regulate the spin-up rate and to minimize the compound pendulum mode under the fixed tether length. The satellite encountered a sticky spot on the flat floor around 60-70 second interval, but shows a robust response back to the target angular rate. The constant torque actuation at the steady state indicates an existence of the surface friction which was not considered for linear modeling.

\section{NONLINEAR CONTROL AND EXPERIMENTAL RESULTS}

Even though the gain-scheduled LQR controller performed well for a regulation problem, The linear control performance can be problematic for a tracking purpose. In addition, the transient response until the states reach the steady-state is unsatisfactory. More smooth transient responses are desired. Furthermore, we can explore 

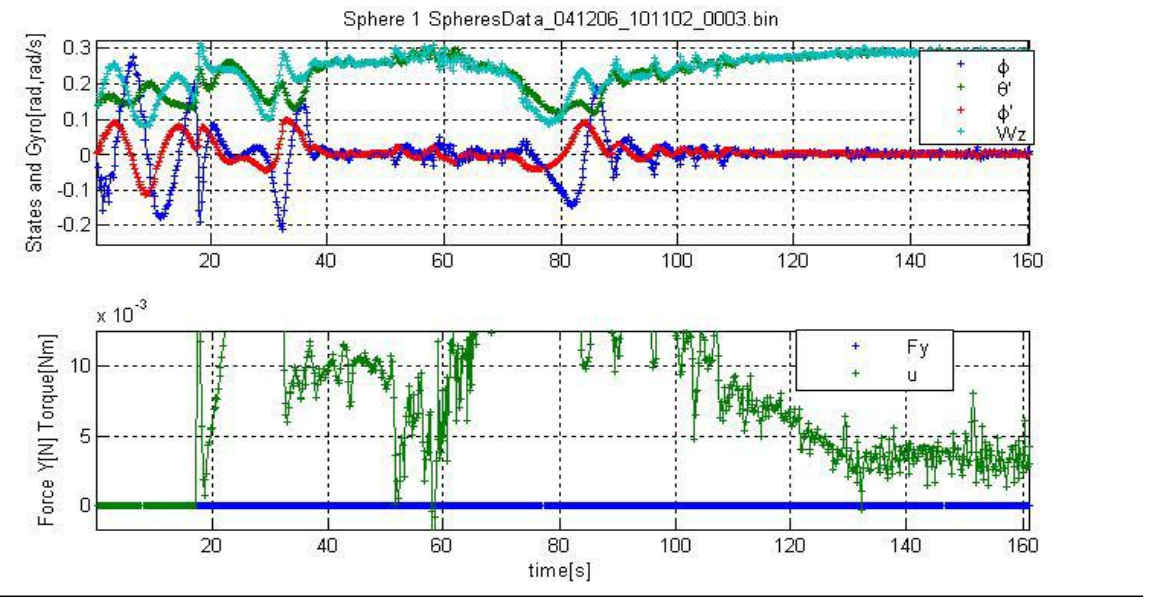

Figure 9. Gain-scheduled LQR controller in a singled-tethered system

the effects of unmodeled dynamics like friction more easily in a nonlinear setup. Therefore, a nonlinear controller fully accounting for the nonlinear dynamics has been developed and implemented in the SPHERES testbed.

\subsection{Input-State Feedback Linearization}

A nonlinear control approach based on Input-State Feedback Linearization ${ }^{11}$ is employed.

A slightly modified form of the nonlinear equation (6) is presented:

$$
[M(q)](\ddot{q})+[C(q, \dot{q})](\dot{q})+[K](\dot{q})=[F]
$$

where $[K]$ is the friction coefficient, $(q)=[\theta ; \phi]$ And the control force $[F]$ is defined as follows:

$$
F=[M(q)] v+[C(q, \dot{q})] \dot{q}+[K](\dot{q})
$$

where $v$ is the new control input.

$$
v=\ddot{q}_{d}-2 \lambda \dot{\tilde{q}}-\lambda^{2} \tilde{q}
$$

where $\tilde{q}=q-q_{d}$. and $\lambda>0$.

Figure 10 shows the experimental data with this nonlinear controller collected from the first SPHERES of a two-body formation. The third row of the figure represents the tether length measured directly using the ultrasound pseudo-GPS metrology system. It is observed that the pendulum mode $(\phi, \dot{\phi})$ gets excited when the tether reels in as predicted in 4.3. Note that the effect of varying the tether length, or the speed, $i$ was not considered in the nonlinear model. So better performance is achievable by taking into account the motor speed $i$. In addition, compared to the linear controller, a more smooth transient response is accomplished. The steady state error appears somewhat larger than that of the linear control results in the previous section. This is because $1 \mathrm{~Hz}$ of control sampling rate was selected for the control while $10 \mathrm{~Hz}$ was used for the linear controller. It is a call for the designer to make to balance between reduction of steady state errors by increasing the control bandwidth, and smoothing of the high frequency disturbances by lowering the bandwidth. The experiments suggest that a lower control bandwidth like $1 \mathrm{~Hz}$ or $2 \mathrm{~Hz}$ perform well at the expense of the modest increase in steady state errors. During the test sessions at the NASA MSFC (see Fig. 11), December 2004, lack of friction model was identified as the main problem of the initial poor performance of the nonlinear controller. After adding this friction model, the performance became satisfactory as shown in the previous plot. 

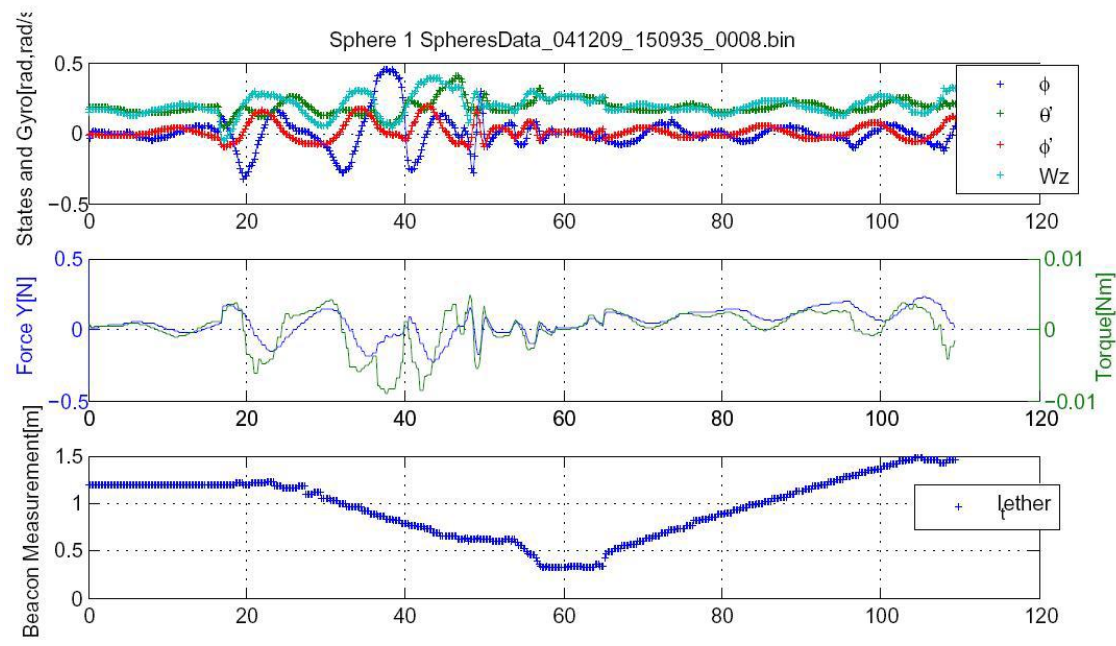

Figure 10. Experiment result of nonlinear controller with varying tether length
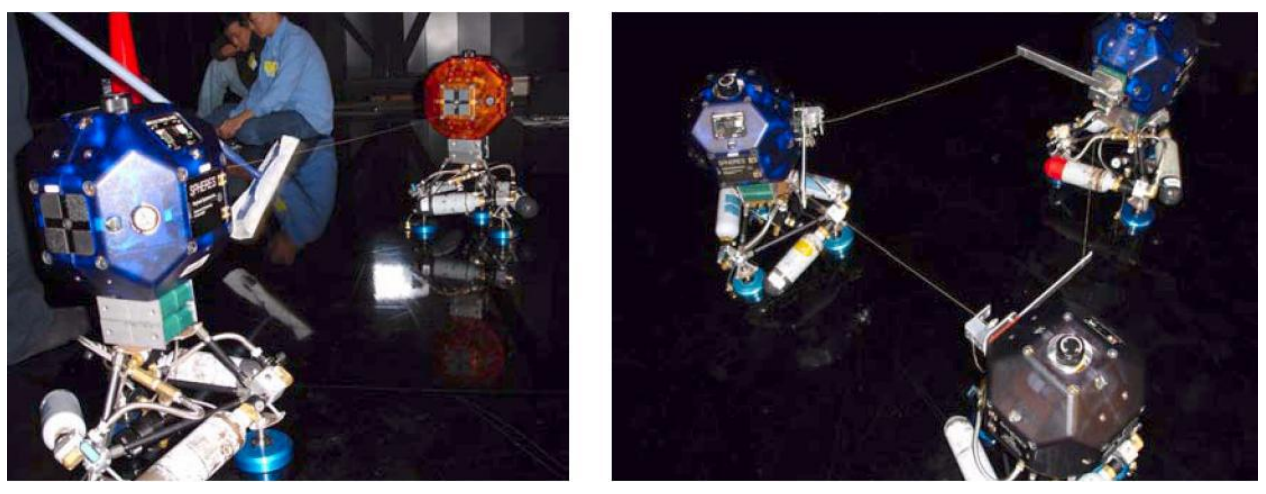

Figure 11. Test on two and triangular formation at the NASA MSFC flat floor 12/10/2004 


\section{CONCLUSION}

The objective of this work is to demonstrate the feasibility of controlling array formation without depending on thrusters for the future space interferometer missions. Linear and nonlinear control techniques only with torque actuators have been successfully implemented into the tethered SPHERES testbed, and tested at the NASA MSFC's flat floor facility using two and three SPHERES configurations. The performance of the LQR controller gain-scheduled over the operating angular rate using only torques was somewhat unsatisfactory resulting in a large pendulum mode angle. The nonlinear control using input-state feedback linearization technique performed well in both two SPHERES in-line configuration and three triangular configuration with varying the tether length. The performance of the nonlinear controller was improved by adding the surface friction model obtained from the previous experiments data. The relative metrology system, using the pseudo-GPS system and the inertial sensor as well as decentralized nonlinear estimator, is developed to provide necessary state information. We are currently updating the testbed with RWA and more sophisticated bearing angle measurement system with force-torque sensor. The video clips of the experiments in this paper can be downloaded at http://ssl.mit.edu/spheres/videos.html.

\section{Acknowledgments}

The authors would like to gratefully acknowledge NASA for both financial and technical support for the MITSSL and PSI SPHERES Tether program. This work has been sponsored under NASA Phase II SBIR contact (Contract No.: NNG05CA09C).

\section{REFERENCES}

1. Mather, J.C et al., "The Submillimeter Frontier: A Space Science Imperative," Astrophysics 1998, astro$\mathrm{ph} / 9812454$.

2. Nolet, S., Kong, E., and Miller, D.W., "Autonomous docking algorithm development and experimentation using the SPHERES testbed," SPIE Defend and Security Symposium, 1315 April 2004, Orlando, FL.

3. Wilson, J.M., Control of a Tethered Artificial Gravity Spacecraft, Ph.D. thesis, Stanford University, 1990.

4. Nakaya, K, et al., "On Formation Deployment for Spinning Tethered Formation Flying and Experimental Demonstration," 18th International Syposium on Space Flight Dynamics, 11-15 October 2004, Munich, Germany.

5. Kokubun, K, et al, "Deployment,Retrieval Control of Tethered Subsatellite Under Effect of Tether Elasticity," J. of Guidance, Control and Dynamics, Vol.19,No.1, January-February 1996.

6. Fujii, H.A., "Wave-Absorbing Control of Transverse Vibration of Tethered System," Journal of Astronautical Sciences, Vol 51, No.3, pp 249-259, 2004.

7. Kim, M., and Hall, C.D., "Control of a Rotating Variable-Length Tethered System," J. of Guidance, Control and Dynamics, Vol.27, No.5, Sep-Oct 2004.

8. Bombardelli, C., Lorenzini, E.C., Quadrelli, M. B., "Retargeting Dynamics of a Linear Tethered Interferometer," AIAA J. of Guidance, Control and Dynamics, Vol. 27, No.6, Nov-Dec 2004.

9. Kumar, K.D. and Yasaka, Y., "Rotating Formation Flying of Three Satellites Using Tethers," J. Spacecraft and Rockets, Vol. 41, No. 6, Nov-Dec 2004.

10. Chung, S.-J., Kong, E. M, and Miller D. W., "Dynamics and Control of Tethered Formation Flight Spacecraft Using the SPHERES Testbed," 2005 AIAA Guidance, Navigation and Control Conference, San Francisco, August 2005.

11. Slotine, J.-J. and Li, W., Applied Nonlinear Control, Prentice Hall. 